\title{
Influence of Cleavage of Photosensitive Group on Thermally Induced Micellization and Gelation of a Doubly Responsive Diblock Copolymer in Aqueous Solutions: A SANS Study
}

\author{
Lilin $\mathrm{He}^{1^{*}}$, Bin $\mathrm{Hu}^{2}$, Daniel M. Henn ${ }^{2}$, and Bin Zhao ${ }^{2}$ \\ ${ }^{1}$ Biology and Soft Matter Division, Oak Ridge National Laboratory, Oak Ridge, Tennessee \\ 37831, USA \\ ${ }^{2}$ Department of Chemistry, University of Tennessee, Knoxville, Tennessee, 37996, USA \\ * Corresponding author. Tel: +1 865576 7748. Email: hel3@ornl.gov
}

This manuscript has been authored by UT-Battelle, LLC under Contract No. DE-AC05O0OR22725 with the U.S. Department of Energy. The United States Government retains and the publisher, by accepting the article for publication, acknowledges that the United States Government retains a non-exclusive, paid-up, irrevocable, world-wide license to publish or reproduce the published form of this manuscript, or allow others to do so, for United States Government purposes. The Department of Energy will provide public access to these results of federally sponsored research in accordance with the DOE Public Access Plan(http://energy.gov/downloads/doe-public-access-plan). 


\begin{abstract}
In the present study, small-angle neutron scattering (SANS) was used to investigate the temperature and concentration dependence of micelle formation and growth of a thermo- and light-responsive hydrophilic block copolymer and the influence of removing photosensitive group. The water-soluble polymer poly(ethylene oxide)-b-poly(ethoxytri(ethylene glycol) acrylate-co-o-nitrobenzyl acrylate) (PEO- $b$-P(TEGEA-co-NBA) underwent sol-gel/gel-sol transitions in response to temperature changes in the range of 15 to $55^{\circ} \mathrm{C}$ in a $25 \mathrm{wt} \%$ aqueous solution. The SANS data showed that individual chains existed at low temperatures and dilute solutions. Spherical micelles formed and packed into an ordered structure at elevated temperatures and high concentrations. Data fitting revealed that the micelle growth followed both micelle fusion and unimer insertion mechanisms after the critical micelle temperature was reached in moderately concentrated solutions whereas the fusion mechanism was dominant at higher temperatures. The UV exposure led to a $32 \%$ expansion in chain dimension at the unimer stage due to repulsive interactions between the ionized segments after the removal of the light sensitive group. The higher onset temperature of sol-gel transition after irradiation was mainly ascribed to the higher lower critical solution temperature (LCST). On the other hand, the lower gel-sol transition temperature upon UV exposure was due to the reduction in micelle volume fraction after the cleavage of o-nitrobenzyl group. These results correlated well with the dynamic rheological data.
\end{abstract}

Key words: Small-angle neutron scattering, Stimuli-responsive polymer, Micellization and Gelation

\title{
1. Introduction
}

Over the past decades, stimuli-responsive polymers have attracted increasing attention because of their potential diverse applications in nanoscience, nanotechnology, and nanomedicine [1]. These smart materials are capable of changing their structures and physical properties in response to external environmental stimuli such as optical, electrical, thermal, mechanical, $\mathrm{pH}$, ionic and magnetic signals [1-7]. Among these materials, block copolymers consisting of two or more chemically distinct polymer components covalently bonded to one 
another are of particular interest due to tunable size and shape of microstructures afforded by simply varying molecular parameters such as chain length and rigidity, as well as interactions between monomeric units and interactions between polymers and surrounding media.

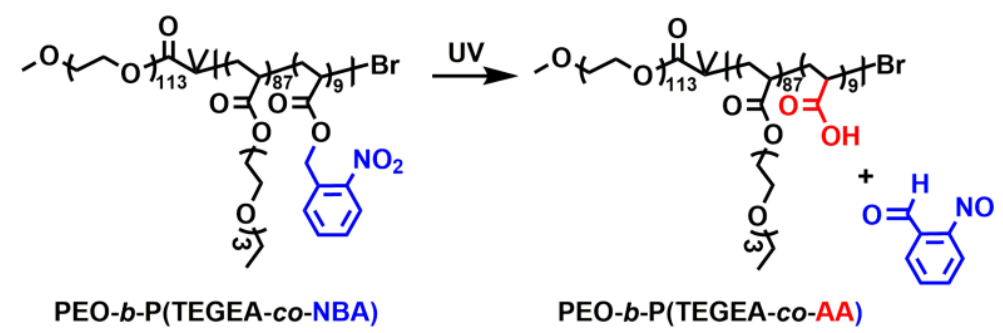

Scheme 1. Thermo- and light-responsive PEO- $b$-P(TEGEA-co-NBA) and photocleavage reaction.

By incorporating light-responsive groups into the thermosensitive blocks, a new type of thermo- and light-sensitive water-soluble block copolymers, poly(ethylene oxide)- $b$ poly(ethoxytri(ethylene glycol) acrylate-co-o-nitrobenzyl acrylate) (PEO- $b$-P(TEGEA-co-NBA)) (Scheme 1), has been developed [3,4]. TEGEA exhibits a lower critical solution temperature (LCST) of $36{ }^{\circ} \mathrm{C}$ in water, while the light sensitive $o$-nitrobenzyl group can undergo a photocleavage reaction when exposed to UV light. This polymer has been shown to undergo multiple micellization and dissociation transitions in water in response to temperature changes and UV irradiation [3]. At low temperatures, the block copolymer is dissolved molecularly in water. With the increase of temperature above the LCST of the thermo- and light-sensitive P(TEGEA-co-NBA) block, micelles form with the dehydrated P(TEGEA-co-NBA) block associated into the core and the PEO block forming the corona. When the concentration is sufficiently high, multiple sol-gel-sol transitions can be achieved. Upon UV irradiation, the hydrophobic $o$-nitrobenzyl group is cleaved, causing the increase of the LCST of the thermosensitive block and the shifts of the sol-gel transition temperatures, which have been seen by vial inversion tests and rheological measurements on a $20.0 \mathrm{wt} \%$ aqueous solutions before and after UV irradiation [4]. Understanding the mechanism of the chains self-assembling into micelles and the influence of removing the light sensitive group on the solution assemblies requires a detailed microstructure characterization of the polymer solutions under various conditions, which is the aim of the present work. 
Among the widely used experimental approaches for microstructure characterization of block copolymers [8-10], SANS is unique and powerful to probe correlations over length scales ranging from a few and several thousand angstroms because the low energies $(\sim 10 \mathrm{meV})$ of cold neutrons avoid radiation damage when interacting with soft matter systems [9]. More importantly, the large contrast between the bound neutron scattering length of hydrogen $(\mathrm{H})$ and deuterium ( $\mathrm{D}$ or ${ }^{2} \mathrm{H}$ ) makes it possible to enhance spatial information by varying the coherent scattering length density (SLD) of the solvent continuously and/or selectively deuterating one particular component without changing the intrinsic properties of the targeted systems. In SANS approach, however, quantitative data analysis frequently requires a suitable model that correctly describes the studied particles. In the SANS investigations of micelles formed by amphiphilic polymers, Pedersen and Gerstenberg developed a parametrized scattering function for the micelles consisting of a homogeneous core surrounded by a corona that contains non-interacting Gaussian chains in dilute solutions following the initial modelling approach proposed by Hayter using spherical form factor and hard-sphere structure factor [11]. Later a more advanced model with self-avoiding interacting chains was developed by Pedersen, including the size polydispersity and instrumental smearing in the fitting as well [12-17]. Recently, Hammouda applied spherical core-shell model with effective structure factor describing particle interaction in the SANS data analysis of Pluronic P85 [18]. In this analysis approach, particle sizes and scattering length densities were directly obtained through data fitting. The aggregation number and the number of hydration water molecules in the shell region were determined through material balance calculations based on the fitting parameters. This analysis method was later used by Martin et al. to determine the distributions of protein and RNA in virus particles [19]. The method with slight modifications was adopted in the present work.

In order to achieve a thorough understanding of the formation and structural evolution of micelles and micellar gels with the change of concentration and temperature and the removal of light sensitive group, we conducted an extensive SANS study of PEO- $b$-P(TEGEA-co-NBA) in $\mathrm{D}_{2} \mathrm{O}$ at concentrations of $0.02,0.2,1,5$, and $25 \mathrm{wt} \%$ before and after the removal of $o$ nitrobenzyl group in a wide temperature window ranging from 15 to $55^{\circ} \mathrm{C}$. Through data fitting using a polydispersed core-shell (PDCS) form factor, hard sphere interaction structure factor and material balance calculations, we have extracted the information on structures of micelles and micellar gels of the block copolymer at different conditions. The key parameters, including the 
aggregation number, micelle volume fraction, degree of hydration, size and scattering length density of each layer, provide insights into the mechanism through which the polymer chains self-organize in response to temperature, concentration and UV exposure. In addition, the correlation between the scattering characteristics and the rheological properties of the micellar samples is discussed.

\section{Experimental}

\subsection{Materials}

The thermo- and light-responsive diblock copolymer PEO- $b$-P(TEGEA-co-NBA) was synthesized by atom transfer radical polymerization following the procedure described in the Supporting Information (SI). The number average molecular weight of $21700 \mathrm{~g} / \mathrm{mol}$ was determined by gel permeation chromatography (GPC) relative to polystyrene standards. The polydispersity index, $\mathrm{M}_{\mathrm{w}} / \mathrm{M}_{\mathrm{n}}$, was 1.22. The degree of polymerization (DP) of the PEO block was 113 while it was 96 for the other block, and the numbers of TEGEA and NBA monomer units were 87 and 9 , respectively (calculated from ${ }^{1} \mathrm{H}$ NMR). Polymer solutions were prepared gravimetrically by dissolving the polymer in $\mathrm{D}_{2} \mathrm{O}$ that was purchased from Cambridge Isotope Inc. The photocleavage of the $o$-nitrobenzyl group was done by exposing the solution samples to long wavelength $(365 \mathrm{~nm}) \mathrm{UV}$ light as described in SI [4]. The unirradiated and irradiated samples were then studied by rheological measurements and dynamical light scattering. The results are shown in the SI.

\subsection{SANS Measurements}

The SANS experiment was conducted at the General Purpose SANS instrument at the High Flux Isotope Reactor, Oak Ridge National Laboratory [20]. Quartz cells with a path length of 2 $\mathrm{mm}$ were used, and the temperature was controlled with a Peltier controller that is able to accurately control the temperature better than one degree. Five concentrations, 0.02, 0.2, 1.0, 5.0 and $25 \mathrm{wt} \%$ were prepared and the scattering data were collected at 15, 27, 30, 35, 43, 52, and $55{ }^{\circ} \mathrm{C}$ after the samples were equilibrated. The neutron wavelength, $\lambda$, was set at $4.72 \AA$ with a wavelength spread, $\Delta \lambda / \lambda$, of 0.13 . Scattered neutrons were detected with a $1 \times 1 \mathrm{~m}^{2}$ twodimensional position sensitive detector. Two sample-to-detector distances, $2.5 \mathrm{~m}$ and $19.3 \mathrm{~m}$, were used to cover a q-range between $q_{\min }=0.003 \AA^{-1}$ and $q_{\max }=0.5 \AA^{-1}$. The raw 2D data 
were corrected for the detector pixel response and dark current, which represents the ambient radiation background and electronic noise, before subtracting the water background. The corrected data were azimuthally averaged to produce the $1 \mathrm{D}$ profile $\mathrm{I}(\mathrm{q})$ vs. $\mathrm{q}$, where $\mathrm{q}=4 \pi$ $\sin \theta / \lambda$ and $2 \theta$ is the scattering angle. Data were placed on an absolute scale $\left(\mathrm{cm}^{-1}\right)$ through the use of calibrated standards [21]. The reduced 1D profiles from the two detector distances were merged using the methods developed by the National Institute of Standards and Technology and implemented in IGOR Pro 6.1 (WaveMetrics, Lake Oswego, OR) [22]. The data were reduced using Igor Pro package provided by the instrument team. Scattering length densities (SLDs) were calculated using the SLD calculator provided by NCNR (http://www.ncnr.nist.gov/resources/index.html). The SLDs for each block of PEO- $b$-P(TEGEAco-NBA) and $\mathrm{D}_{2} \mathrm{O}$ are summarized in Table 1.

Table 1. Scattering length densities (SLD) for each block of PEO- $b$-P(TEGEA-co-NBA) and PEO- $b$-P(TEGEA-co-AA)

\begin{tabular}{lccccc}
\hline & $\begin{array}{c}\text { Chemical } \\
\text { formula }\end{array}$ & $\begin{array}{c}\text { Density } \\
\left(\mathrm{g} / \mathrm{cm}^{3}\right)\end{array}$ & $\begin{array}{c}\text { Molecular } \\
\text { volume }\left(\AA^{3}\right)\end{array}$ & $\begin{array}{c}\text { Scattering } \\
\text { lengths }(\AA)\end{array}$ & SLD $\left(\AA^{-2}\right)$ \\
\hline EO & $\mathrm{C}_{2} \mathrm{H}_{4} \mathrm{O}$ & 1.13 & 64.91 & $4.14 \times 10^{-5}$ & $0.64 \times 10^{-6}$ \\
TEGEA & $\mathrm{C}_{11} \mathrm{H}_{20} \mathrm{O}_{5}$ & 1.03 & 37.45 & $2.73 \times 10^{-4}$ & $0.73 \times 10^{-6}$ \\
$\mathrm{NBA}$ & $\mathrm{C}_{10} \mathrm{H}_{9} \mathrm{O}_{4} \mathrm{~N}$ & 1.20 & 97.30 & $6.54 \times 10^{-4}$ & $2.28 \times 10^{-6}$ \\
$\mathrm{AA}$ & $\mathrm{C}_{3} \mathrm{H}_{4} \mathrm{O}_{2}$ & 1.05 & 68.63 & $1.66 \times 10^{-4}$ & $1.46 \times 10^{-6}$ \\
$\mathrm{D}_{2} \mathrm{O}$ & $\mathrm{D}_{2} \mathrm{O}$ & 1.11 & 29.97 & $1.91 \times 10^{-4}$ & $6.37 \times 10^{-6}$ \\
\hline
\end{tabular}

\subsection{SANS Data Analysis Using Spherical Core-Shell Model and Material Balance Calculation}

The scattering form factor and structure factor for a monodispersed system can be written as $[9,23]:$

$$
I(q)=\emptyset V_{p}(\Delta \rho)^{2} P(q) S(q)+I_{\text {inc }}(1)
$$

where $\emptyset$ is the volume fraction of micelles and $V_{p}$ is the particle volume. $\Delta \rho^{2}$ is the contrast factor; $P(q)$ is the form factor and $S(q)$ is the structure factor. $I_{\text {inc }}$ arises from the incoherent scattering background of the samples.

The PDCS model is used to describe the spherical micelles in the solutions at high temperatures. In this model, the core is predominantly hydrophobic. Water and a small fraction 
of hydrophilic component are also allowed in this region. The corona contains the remaining hydrophilic blocks and water. The SANS intensity is given by [24]:

$$
I(q)=n\left(\frac{3 V_{c}\left(\rho_{c-} \rho_{S}\right) j_{1}\left(q R_{c}\right)}{q R_{c}}+\frac{3 V\left(\rho_{s-} \rho_{\text {water }}\right) j_{1}(q R)}{q R}\right)^{2} S(q)+I_{\text {inc }}
$$

Where $n$ is micelle number density, it relates to $\emptyset$ via $n=\emptyset / V . V_{c}$ and $V$ are the volumes of core and the whole particle, respectively. $R_{c}$ and $R$ are the core radius and total radius of the particle, $\rho_{c}$ and $\rho_{s}$ are the SLDs of core and shell, respectively. Note that $V_{i}=\left(\frac{4 \pi}{3}\right) R_{i}^{3}$ and $j_{1}(x)=(\sin x-x \cos x) / x^{2}$ is the spherical Bessel function of the first kind. The structure factor $S(q)$ of the spherical micelles is estimated using the Percus-Yevick approximation for hard spheres in the Ornstein-Zernicke equations [25,26]. The instrument smearing and size polydispersity are also taken into account in our data fitting [9]. The size of the particle follows a Schulz distribution. The fitting is completed using Igor Pro's built in non- linear least squares routine $[9,22]$. Once the size and SLD of each layer are obtained, we use the material balance equations to gain further structural information as a function of temperature for the well-defined micelles formed in the high concentration (25 wt\%) [18]

$$
\begin{gathered}
N_{a g g}\left(87 \cdot v_{T E G E A}+9 \cdot v_{N B A \text { or } A A}+113 \cdot v_{E O} \cdot f\right)+n_{1} \cdot v_{D_{2} O}=\frac{4 \pi}{3} R_{C}^{3} \\
N_{a g g}\left(113 \cdot v_{E O} \cdot(1-f)\right)+n_{2} \cdot v_{D_{2} O}=\frac{4 \pi}{3}\left(R^{3}-R_{C}^{3}\right) \\
N_{a g g}\left(87 \cdot b_{T E G E A}+9 \cdot b_{N B A \text { or } A A}+113 \cdot b_{E O} \cdot f\right)+n_{1} \cdot b_{D_{2} O}=\rho_{C} \cdot \frac{4 \pi}{3} R_{C}^{3} \\
N_{a g g}\left(113 \cdot b_{E O} \cdot(1-f)\right)+n_{2} \cdot b_{D_{2} O}=\rho_{s} \cdot \frac{4 \pi}{3}\left(R^{3}-R_{C}^{3}\right)
\end{gathered}
$$

where $N_{a g g}$ is the aggregation number of the polymer in each micelle. $v_{T E G E A}, v_{N B A}$ or AA,$v_{E O}$, and $v_{D_{2} O}$ are the monomer volumes of TEGEA, NBA (AA after the $o$-nitrobenzyl group is removed), EO and $\mathrm{D}_{2} \mathrm{O}$, respectively. $b_{T E G E A}, b_{N B A}$ or $A A, b_{E O}$ and $b_{D_{2} O}$ are the scattering lengths, respectively. The monomer volume and scattering length of each monomer unit are listed in Table 1. There are 113 EO monomer units, 87 TEGEA and 9 NBA or AA monomer units per chain. $f$ denotes the fraction of the EO blocks in the micelle core. Different than the parameters $y_{a}$ and $y_{b}$ that were defined as the number of $\mathrm{D}_{2} \mathrm{O}$ molecules per EO monomer in Hammouda's 
original paper [18], $n_{1}$ and $n_{2}$ in our equations are the number of $\mathrm{D}_{2} \mathrm{O}$ in the core and corona, respectively. Solving the linear equations gives us $N_{a g g}, f, n_{1}$ and $n_{2}$.

\section{Results and Discussion}

\subsection{Thermally Induced Micellization of PEO-b-P(TEGEA-co-NBA) Before and After UV} Irradiation in 0.02 wt\% Aqueous Solution

The polymer solution exhibited unimer state at low weight fractions and below its critical micellization temperature (CMT). Figure 1 shows the SANS profiles for 0.02 wt \% solutions of PEO- $b$-P(TEGEA- $c o-N B A)$ and PEO- $b$-P(TEGEA-co-acrylic acid) (PEO- $b$-P(TEGEA-co-AA), the polymer formed after the UV irradiation of PEO- $b$-P(TEGEA-co-NBA)) in $\mathrm{D}_{2} \mathrm{O}$, at $15{ }^{\circ} \mathrm{C}$. The profiles are similar to those of P85 Pluronic and PEO in $\mathrm{D}_{2} \mathrm{O}$ at low temperatures [18,27]. The low-q upturn could be attributed to loosely-assembled clusters [18]. Fitting the scattering curves using the approximate analytical model for polymers with excluded volume effects yields the polymer radius of gyration $R_{g}$ and Porod exponent [28]:

$$
I(q)=\frac{1}{v U^{\frac{1}{2 v}}} \gamma\left(\frac{1}{2 v}, U\right)-\frac{1}{v U^{\frac{1}{v}}} \gamma\left(\frac{1}{v}, U\right)
$$

where $\gamma(x, U)$ is the incomplete Gamma function:

$$
\gamma(x, U)=\int_{0}^{U} d y \exp (-y) y^{x-1}
$$

and

$$
U=\frac{q^{2} R_{g}^{2}(2 v+1)(2 v+2)}{6}
$$

where $v$ is the excluded volume parameter that is related to the Porod exponent $m$ as $v=1 / m$. A Porod exponent 1.94 at high q region indicates that the chains of unirradiated sample adopt a nearly ideal conformation. In contrast, a smaller exponent 1.59 of irradiated sample implies relatively stretched chains due to stronger repulsive interactions between monomer units. This agrees with our previous observation of a noticeable decrease in the solution $\mathrm{pH}$ after the cleavage of $o$-nitrobenzyl groups by UV irradiation [3]. The pendant $-\mathrm{COOH}$ groups, form after the photochemical reaction, ionize to a certain degree, resulting in charges on the polymer chain and electrostatic repulsive interactions. $R_{g}$ increases from $25.5 \AA$ to $31.1 \AA$ upon UV-induced cleavage of $o$-nitrobenzyl group, which confirms that charge repulsion leads to an expansion of overall dimension of chains. 


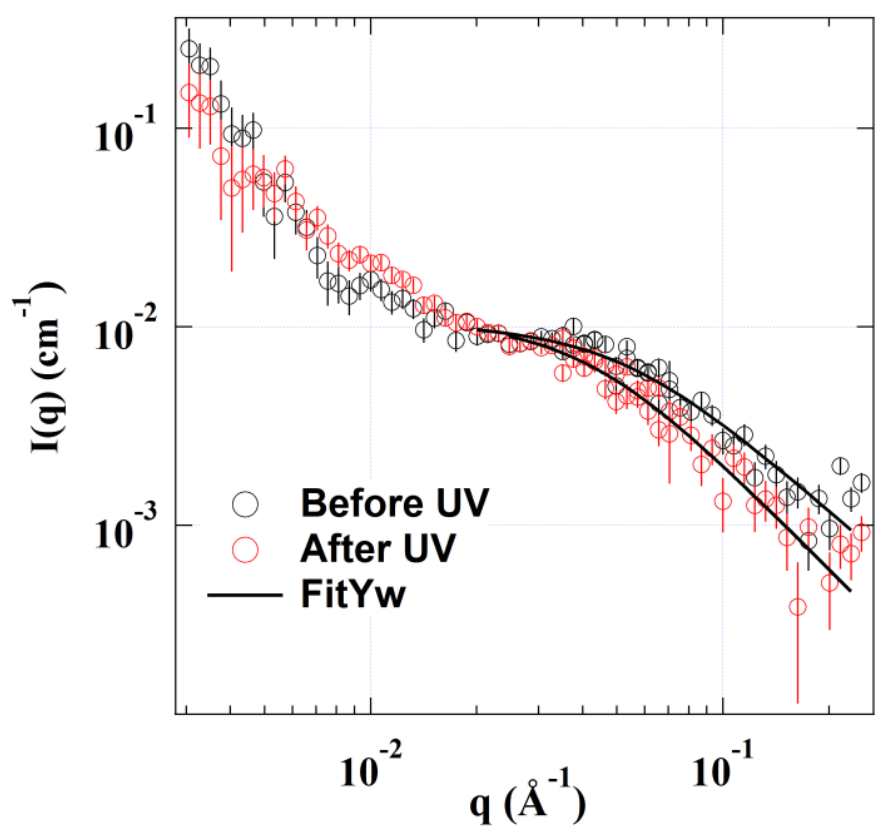

Figure 1. SANS profiles for $0.02 \mathrm{wt} \%$ solutions of (०) PEO- $b$-P(TEGEA-co-NBA) and (०) PEO- $b$ - $\mathrm{P}$ (TEGEA-co-AA) in $\mathrm{D}_{2} \mathrm{O}$ at $15{ }^{\circ} \mathrm{C}$. The flat incoherent scattering background is subtracted. The fits of the high $q$ part of the curves using approximate analytical model for polymer with excluded volume effects are shown as solid lines.

When the temperature was raised to $27^{\circ} \mathrm{C}$, above the CMT of the polymer, the P(TEGEA-coNBA) block became more hydrophobic and collapses to form micelle core whereas the chain property of PEO was not significantly influenced in this temperature range $[3,4,29]$. This is evidenced by a dramatic increase in the SANS intensity of $0.02 \mathrm{wt} \%$ solution from 15 to $27{ }^{\circ} \mathrm{C}$ (Fig. 2 left). Note that we previously measured the critical micelle concentrations (CMCs) of two doubly responsive diblock copolymer samples with similar numbers of monomer untis in the second block, $\mathrm{PEO}_{113}-b-\mathrm{P}\left(\mathrm{TEGEA}_{70}-c o-\mathrm{NBA}_{9}\right)$ and $\mathrm{PEO}_{113}-b-\mathrm{P}\left(\mathrm{TEGEA}_{109}-c o-\mathrm{NBA}_{14}\right)$ where the subscripts stand for numbers of monomer units, by fluorescence spectrometry, and they were $0.064 \mathrm{mg} / \mathrm{mL}$ at $32{ }^{\circ} \mathrm{C}$ and $0.025 \mathrm{mg} / \mathrm{mL}$ at $30{ }^{\circ} \mathrm{C}$, respectively [3]. DLS study showed that the unimer-micelle transition of the polymer at the $0.05 \mathrm{wt} \%$ concentration before UV irradiation (see S2 in SI) took place at $28^{\circ} \mathrm{C}$, in a good agreement with the SANS measurements, though the concentrations in DLS and SANS experiments were slightly different. The low- $q$ upturn gradually disappears with the formation of micelles, suggesting that the polymer chains in the 
large loose clusters reorganized into micelles. The scattering intensity decays following $q^{m}$ with $m$ close to -1 in the low- $q$ region at $27{ }^{\circ} \mathrm{C}$. Failure of fitting data to any single model indicates coexistence of considerable amount of unimers and micelles [30]. The micelles grow with increasing temperature, which eventually is characterized by an almost flat signal in the low $\mathrm{q}$ region and $1 / q^{4}$ decay in the intermediate- $q$ Porod region. No substantial structural change was observed above this temperature. Upon UV exposure, the scattering intensity exhibited different temperature dependence from the unirradiated sample. First of all, the micellization occurred between 35 and $43{ }^{\circ} \mathrm{C}\left(33{ }^{\circ} \mathrm{C}\right.$ observed by DLS), a significantly higher temperature than that of the unirradiated sample. The removal of the $o$-nitrobenzyl group increases the hydrophilicity of the thermosensitive block and thus the LCST, and more energy is required to transfer chains to form micelles. Therefore, the onset temperature of the micellization is raised. No significant change was observed beyond $52{ }^{\circ} \mathrm{C}$. A slope between -2 and -3 in the intermediate $\mathrm{q}$ region indicates no well-defined interface between the micelles and surrounding media (Fig. 2 right).
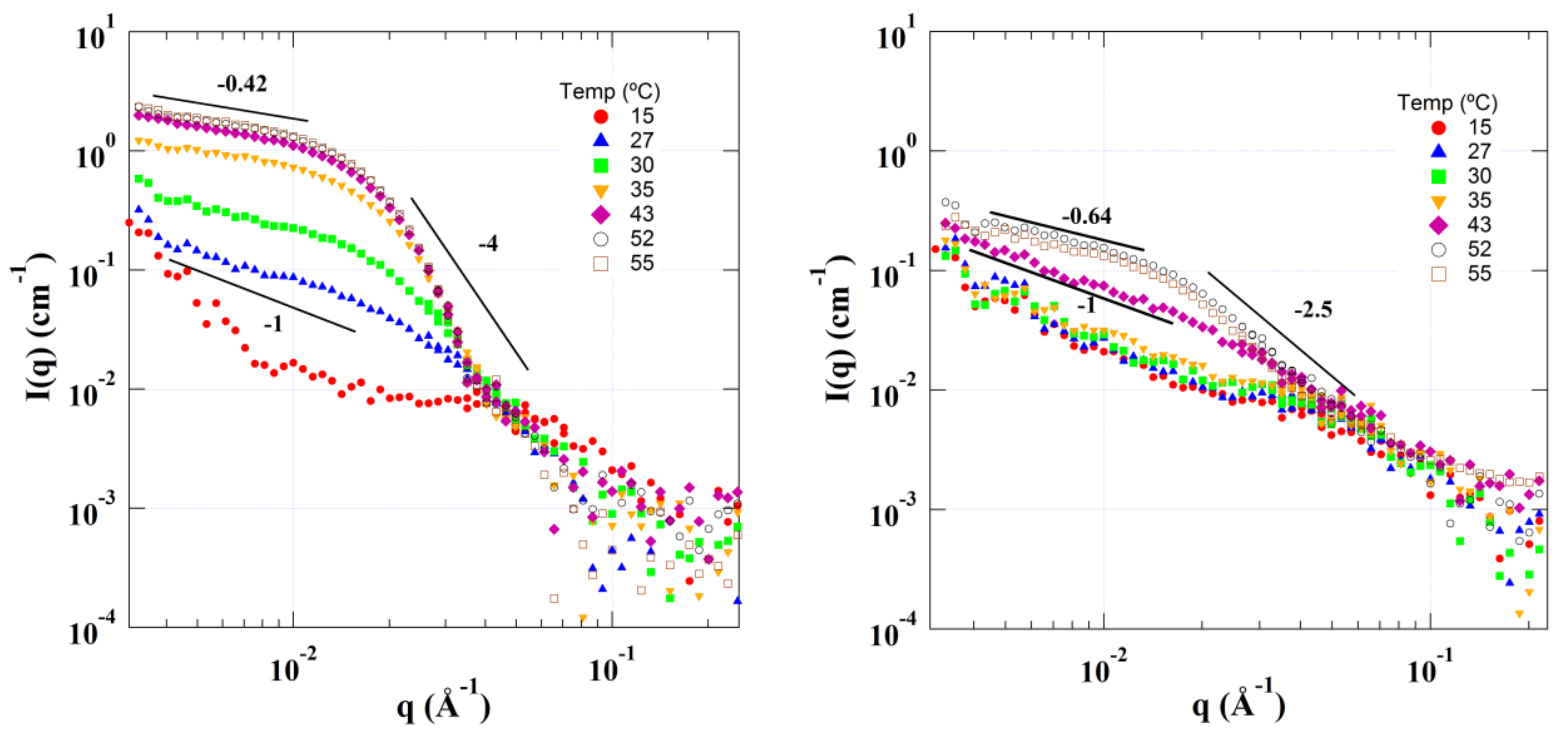

Figure 2. SANS profiles from 0.02 wt \% solutions (left) PEO- $b$-P(TEGEA-co-NBA) and (right) PEO- $b-\mathrm{P}\left(\right.$ TEGEA-co-AA) as a function of temperature in $\mathrm{D}_{2} \mathrm{O}$. The flat background is subtracted. The error bars on the SANS curves are not added for the sake of clarity. The scattering slopes are plotted in the graph as a guide to the eye. 

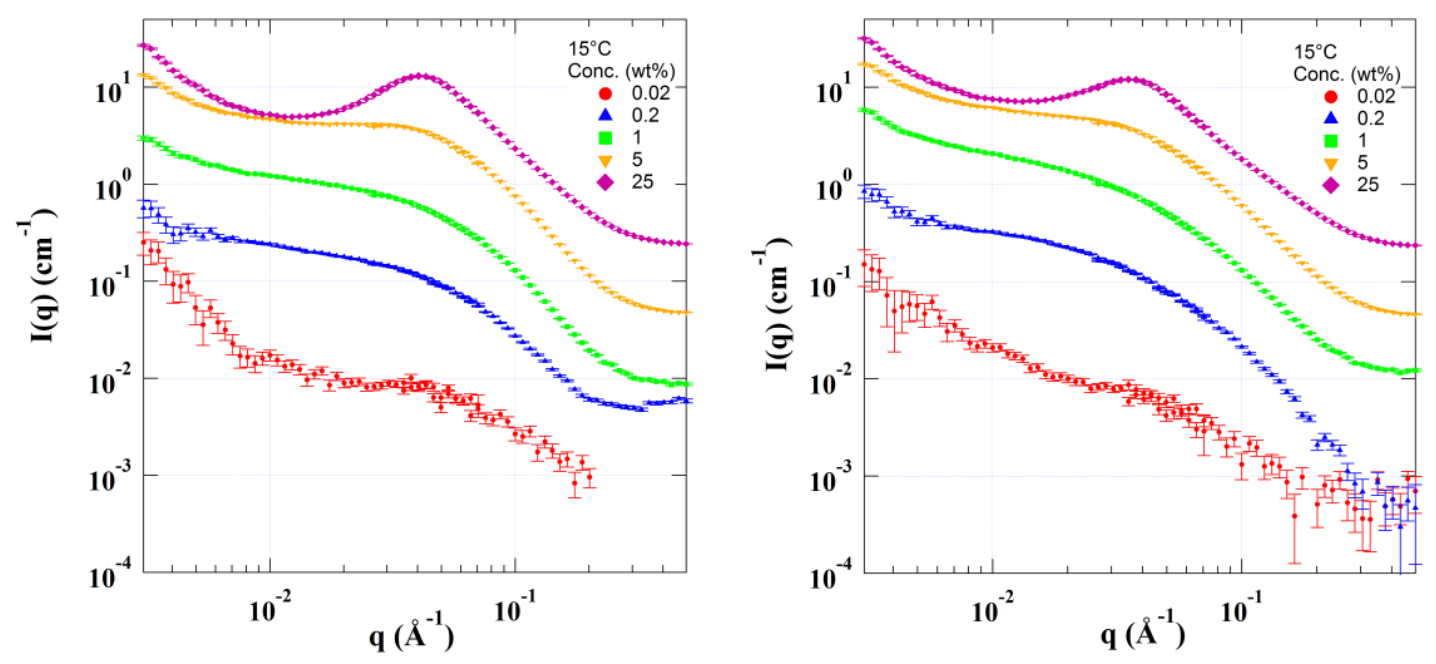

Figure 3. SANS profiles before (left) and after UV exposure (right) at $15^{\circ} \mathrm{C}$ for concentrations between $0.02 \mathrm{wt} . \%$ and $25 \mathrm{wt} \%$. The different flat incoherent backgrounds are attributed to different concentrations.

3.2 Effect of Polymer Concentration on Solution State of Block Copolymer at $15{ }^{\circ} \mathrm{C}$ and $55{ }^{\circ} \mathrm{C}$ Before and After UV Irradiation

SANS profiles of both irradiated and unirradiated samples at 0.02, 0.2, 1, 5 and $25 \mathrm{wt} \%$ at 15 and $55{ }^{\circ} \mathrm{C}$ are summarized in Figures 3 and 4, respectively. For the unirradiated samples displayed at $15{ }^{\circ} \mathrm{C}$ (left), one notices that the scattering arising from loosely-organized clusters at low q region weakens with increasing concentration from 0.02 to $0.2 \mathrm{wt} \%$ and it then persists even when the concentration reaches $25 \mathrm{wt} \%$. The broad peak at $\sim 0.04 \AA^{-1}$ is ascribed to correlation hole that has no simple physical meaning [31]. Akin to the unirradiated samples, the irradiated samples at $15^{\circ} \mathrm{C}$ follow the same concentration dependence except for more expanded conformation of the chains.

At $55{ }^{\circ} \mathrm{C}$, a temperature well above the LCST of the P(TEGEA-co-NBA) block, the collapse of the thermosensitive component leads to the formation of micelles. The PDCS model with hard sphere interactionsis capable of describing the well-defined particles formed in the samples. The fitting yields information including particle volume fraction, core radius, corona thickness and SLD in each layer. Note that the sizes of core and corona picked up by the model fitting strongly 
depend on the difference in SLD and interface sharpness between core and corona regions. The fitting is shown as solid lines through the data points in Figure 4. At the lowest concentration, the particles are isolated, thus no structure factor is needed in the model. Starting from $0.2 \mathrm{wt} \%$, a broad maximum due to interparticle interactions between $0.01 \AA^{-1}$ and $0.02 \AA^{-1}$ develops and becomes rather sharp for $25 \mathrm{wt} \%$, which is characteristic of well-ordered arrangement of the micelles. The center-to-center distance between neighboring micelles can be obtained using equation: $d \approx 2 \pi / q_{\max }=314 \AA$. For the irradiated samples in the right graph of Figure 4 , the same model is used to fit all the concentrations. For the $25 \mathrm{wt} \%$ solution, compared with the unirradiated sample, a broader and less intense correlation peak is observed in the most concentrated sample indicating a less ordered arrangement. In addition, the change of slope in the low $\mathrm{q}$ region might be attributed to morphological transition, which needs further investigation.
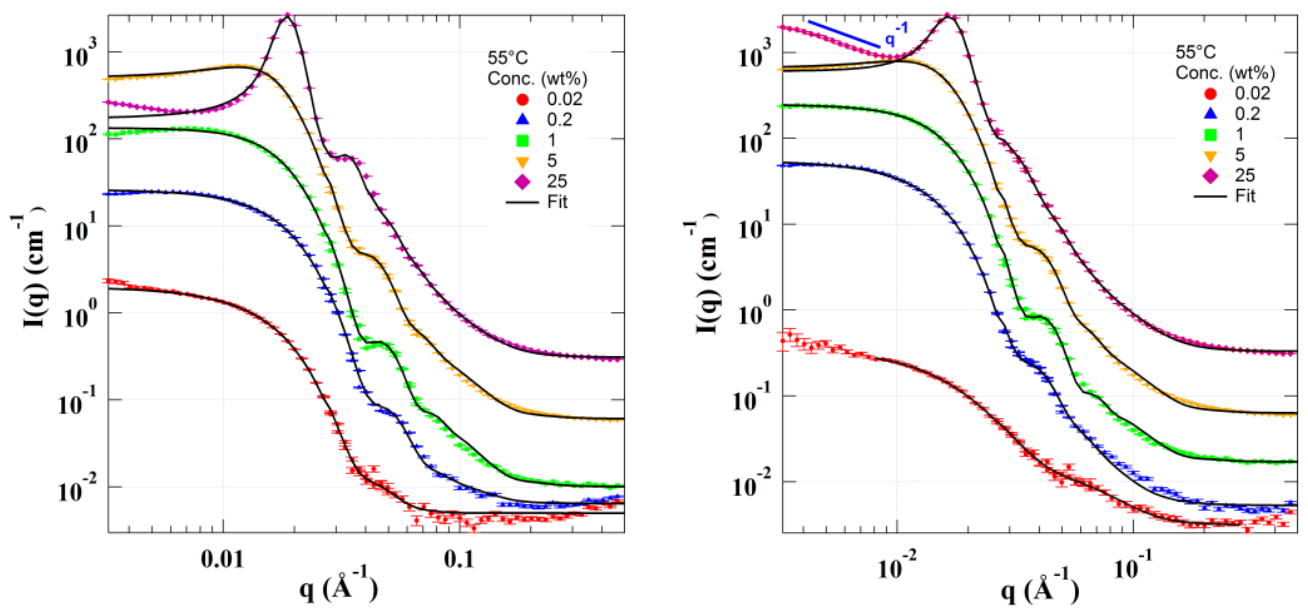

Figure 4. SANS profiles and corresponding model fitting of samples at various concentrations at $55^{\circ} \mathrm{C}$ before (left) and after UV exposure (right). The solid lines correspond to the best fits using the polydispersed core-shell spherical model. The fitting parameters are displayed in Table 2 and 3. The different flat incoherent backgrounds are attributed to different concentrations.

Table 2 and 3 summarize the fitting parameters of the scattering curves using the model at various concentrations at $55^{\circ} \mathrm{C}$ for both unirradiated and irradiated samples, respectively. The size polydispersity of micelles is shown in Table S1 (SI). The micelles at all measured concentrations consist of a large hydrophobic core and relatively thin hydrophilic corona except 
for the most dilute irradiated sample. The total radius of micelles for the unirradiated sample goes up from $134 \AA$ in the 0.02 wt.\% solution to $179 \AA$ in the 25 wt\% solution whereas it increases from $70 \AA$ in the 0.02 wt. \% to the maximum of $203 \AA$ in the 0.2 wt $\%$, then shrinks to $181 \AA$ in the $25 \mathrm{wt} \%$ solution for the irradiated sample.

Note that the core radius of the micelles in the unirradiated samples increases from $108 \AA$ in the 0.02 wt.\% solution to $140 \AA$ in the $25 \mathrm{wt} \%$ solution whereas the SLD declines dramatically with respect to concentration. The total scattering length of the core, calculated according to volume $v$ and SLD $\rho$ via $\sum b=v \cdot \rho$, goes down with increasing concentration. This indicates that $\mathrm{D}_{2} \mathrm{O}$ molecules are progressively replaced by the polymer chains in the core region when the concentration goes up. Significant amount of water molecules still exist in that region, which is suggested by the SLD of the micelle core in the solution of $25 \mathrm{wt} \%$. Compared with the unirradiated sample, a smaller core with lower SLD forms in the irradiated $0.02 \mathrm{wt} \%$ sample, and both parameters increase dramatically when the concentration increased to $0.2 \mathrm{wt} \%$. The core size hardly changes with further increasing concentration. Moreover, the total scattering length of the core for the micelles in the irradiated sample is much smaller than in the unirradiated sample at $25 \mathrm{wt} \%$. This is ascribed to the removal of $o$-nitrobenzyl group that has a larger SLD than other components in the polymer as shown in Table 1. Hence, the scattering data and fitting provide direct evidence that the monomer units in the thermosensitive block are predominantly located in the core and EO monomer units are in the corona.

The corona thickness of the unirradiated sample varies nonmonotonically with concentration at $55^{\circ} \mathrm{C}$ and a maximum is found in the $1.0 \mathrm{wt} \%$ solution whereas the SLD in this region hardly changes. In $0.02 \mathrm{wt} \%$ solution, one notices that the SLD of the corona, $5.89 \times 10^{-6} \AA^{-2}$, and that of the core, $5.15 \times 10^{-6} \AA^{-2}$, are not very different from the SLD of pure $\mathrm{D}_{2} \mathrm{O}$ is $6.37 \times 10^{-6} \AA^{-2}$, indicating that both regions should contain significant amount of water. The difference in SLD between the core and the shell continuously goes up with increasing concentration, reflecting the replacement of water by the collapsed polymer chains in the core region with more chains joining in the micelles. For the micelles formed in the irradiated sample, both thickness and SLD of the corona go up when increasing the concentration from 0.02 wt.\% to $0.2 \mathrm{wt} \%$. The subsequent decrease in both parameters with further increasing concentration reflects the corona shrinkage due to tighter packing. 
Table 2. Fitting results for the samples before UV exposure (PEO- $b$-P(TEGEA-co-NBA)) at 55 ${ }^{\circ} \mathrm{C}$ and various concentrations using the PDCS model.

\begin{tabular}{cccccc}
\hline \multirow{2}{*}{$\begin{array}{c}\text { Concentration } \\
(\mathrm{wt} \%)\end{array}$} & Radius & SLD & Thickness & SLD & Total radius \\
\cline { 2 - 6 } & $(\AA)$ & $\left(\times 10^{-6} \AA^{-2}\right)$ & $(\AA)$ & $\left(\times 10^{-6} \AA^{-2}\right)$ & $(\AA)$ \\
\hline 0.02 & 107.8 & 5.15 & 25.9 & 5.89 & 133.7 \\
0.2 & 107.0 & 4.22 & 43.9 & 6.12 & 150.9 \\
1 & 115.8 & 2.78 & 52.3 & 6.01 & 168.1 \\
5 & 126.8 & 1.72 & 48.6 & 5.95 & 175.4 \\
25 & 139.7 & 1.30 & 39.4 & 5.94 & 179.1 \\
\hline
\end{tabular}

Table 3. Fitting results for the samples after UV exposure (PEO- $b-\mathrm{P}(\mathrm{TEGEA}-\mathrm{co}-\mathrm{AA}))$ at $55^{\circ} \mathrm{C}$ and various concentrations using the PDCS model.

\begin{tabular}{cccccc}
\hline \multirow{2}{*}{$\begin{array}{c}\text { Concentration } \\
(\mathrm{wt} \%)\end{array}$} & Radius & SLD & Thickness & SLD & Total radius \\
\cline { 2 - 6 } & $(\AA)$ & $\left(\times 10^{-6} \AA^{-2}\right)$ & $(\AA)$ & $\left(\times 10^{-6} \AA^{-2}\right)$ & $(\AA)$ \\
\hline 0.02 & 16.2 & 1.18 & 53.6 & 6.15 & 69.8 \\
0.2 & 141.3 & 2.84 & 62.0 & 6.27 & 203.3 \\
1 & 131.0 & 2.04 & 52.7 & 5.86 & 183.7 \\
5 & 136.3 & 1.76 & 49.7 & 5.90 & 186.0 \\
25 & 139.2 & 0.82 & 41.7 & 5.67 & 180.9 \\
\hline
\end{tabular}



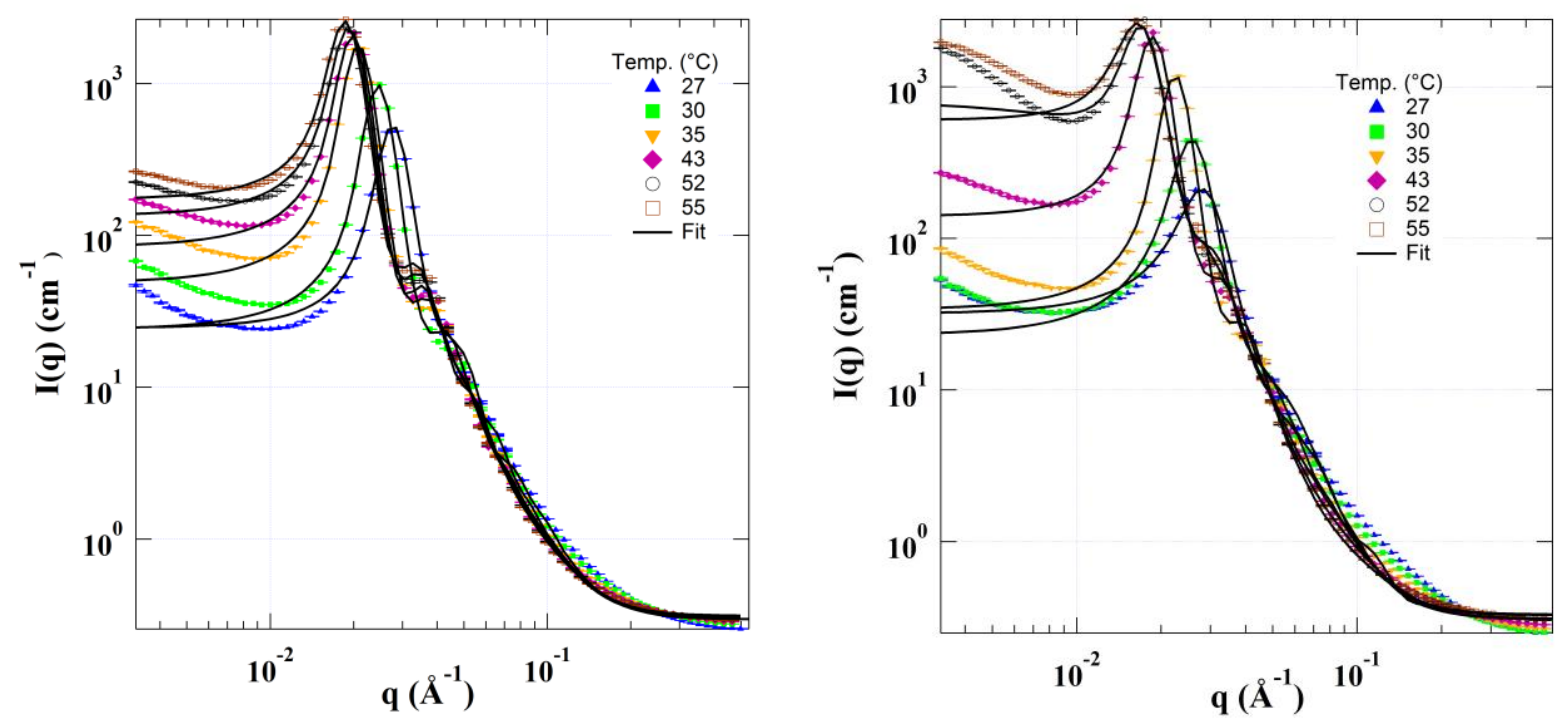

Figure 5. SANS profiles and data fitting using polydispersed core-shell model and hard sphere interaction potential for the most concentrated sample (25 wt\%) before (left) and after UV (right) as a function of temperature. The size polydispersity of micelles obtained from the model fitting is shown in Table $\mathrm{S} 2$ (SI)

\subsection{5 wt\% Aqueous Solution of PEO-b-P(TEGEA-co-NBA) Before and After UV Irradiation}

In order to correlate the structural changes with the viscoelastic properties of these solutions, the SANS data of $25 \mathrm{wt} \%$ solutions at various temperatures were chosen for further analysis. The dynamic viscoelastic properties of the aqueous solution before and after UV irradiation were measured using a constant frequency of $1 \mathrm{~Hz}$ in a heating ramp with a heating rate of $3{ }^{\circ} \mathrm{C} / \mathrm{min}$, which are shown in Figure S1 (Supplementary Information). A strain amplitude of $\gamma=0.2 \%$ was applied. The sol-gel-sol transitions are observed for both samples in the temperature range from 15 to $55^{\circ} \mathrm{C}$. The unirradiated sample exhibits the first transition when the temperature was raised from 25 to $30{ }^{\circ} \mathrm{C}$. The second transition takes place at $54.5^{\circ} \mathrm{C}$. For the irradiated sample, the sharp increase in both $\mathrm{G}^{\prime}$ and $\mathrm{G}^{\prime \prime}$ at $34.8^{\circ} \mathrm{C}$ reflects the first sol-gel transition while the second one occurs at $52.4{ }^{\circ} \mathrm{C}$. Obviously the irradiated sample shows a much narrower hard gel zone than the unirradiated one.

Figure 5 displays the scattering profiles of both samples as a function of increasing temperature and corresponding fitting using the PDCS model. Since the corona regions of neighboring micelles overlap at such high concentration, the model with hard sphere interaction 
no longer properly accounts for micelle-micelle interactions [32,33]. In our case, however, structural information can still be semiquantified by using this model because only less than $10 \%$ of the overall micellar dimension interpenetrates according to the estimation based on the interparticle distance and the particle size at a certain temperature. Enhanced scattering intensity and shift of the first minimum toward smaller q with increasing temperature for both unirradiated and irradiated samples reflect progressive growth of the micelles. The fitting parameters: core radius, corona thickness and volume fraction are plotted as a function of temperature for both samples in Figure 6 and Figure 7. As seen on the graphs, the core radius is larger than the corona thickness for both samples in the whole measured temperature range. Compared to the monotonic increase observed for the unirradiated sample, the core size of micelles in the irradiated sample gradually increases in the temperature range from 27 to $52{ }^{\circ} \mathrm{C}$ followed by a slight drop at higher temperatures for the irradiated sample. The thickness of corona, on the other hand, fluctuates at the low temperatures and reaches maximum at $35{ }^{\circ} \mathrm{C}$ and $43{ }^{\circ} \mathrm{C}$ for the unirradiated and irradiated samples, respectively. The subsequent shrinkage of the corona in both samples is mainly attributed to the loss of water reflecting reduced miscibility of water and PEO chains above $43{ }^{\circ} \mathrm{C}[34]$.
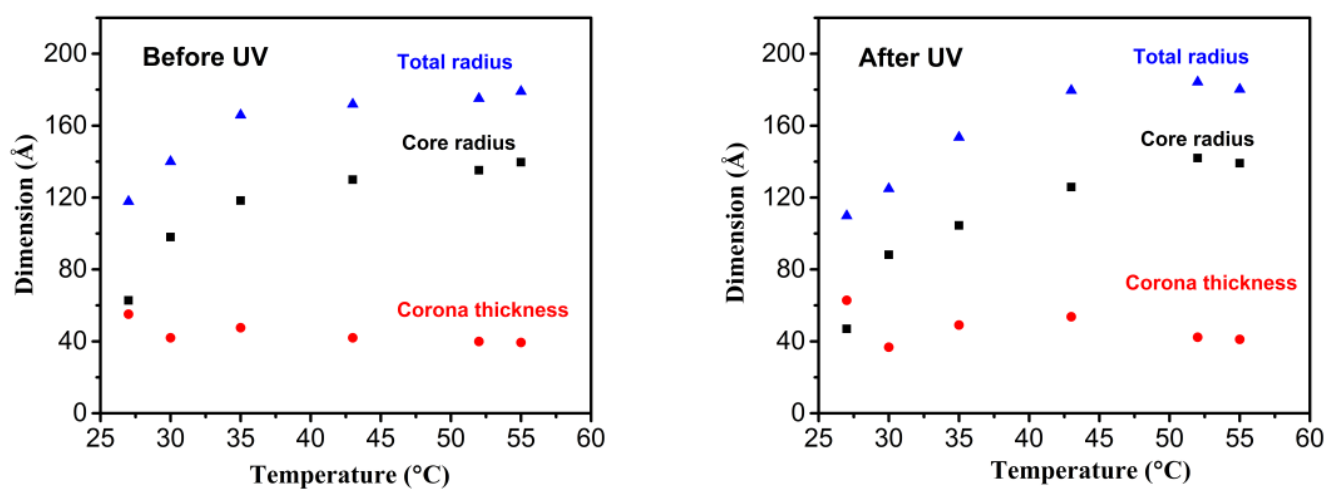

Figure 6. Variation of the core radius, corona thickness and total size of the micelles as a function of temperature for the $25 \mathrm{wt} \%$ samples before (left) and after UV irradiation (right). 


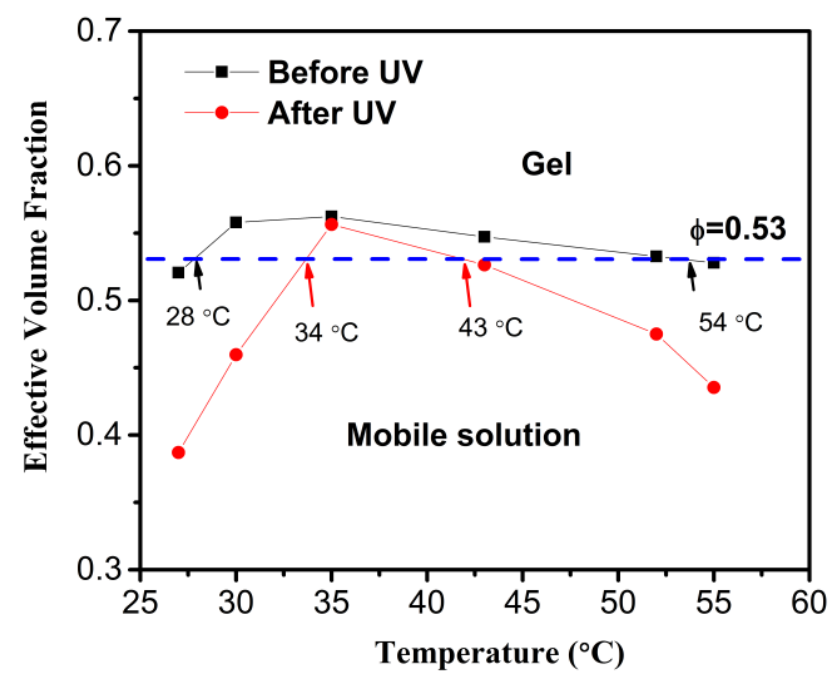

Figure 7. Temperature dependence of effective volume fraction of micelles for aqueous solutions of PEO- $b$-P(TEGEA- $c o-N B A)$ ( $\boldsymbol{n}$ ) and PEO- $b$-P(TEGEA-co-AA) formed from PEO- $b$ $\mathrm{P}($ TEGEA-co-NBA) after UV irradiation $(\bullet)$ at $25 \mathrm{wt} \%$. The broken line denotes the boundary between the mobile solution and the gel. The effective volume fractions of micelles were obtained through the datafitting using equation 1.

The effective volume fraction of micelles, which reflects the tightness of the micelle packing in solution, is one of the key factors to determining the viscoelastic properties of polymer solutions. It is well known that the sample transits from a mobile fluid to a gel once the effective volume fraction of the micelles in the system, $\phi$, reaches a critical value of $0.53 \pm 0.02$ [35]. Following the initial increase, the volume fraction of the micellar particles in the unirradiated sample crosses the $\phi=0.53$ line (the blue broken line in Figure 7) at $28{ }^{\circ} \mathrm{C}$ at which the sol-gel transition occurs. After reaching the maximum volume fraction of 0.56 at $35{ }^{\circ} \mathrm{C}$, it slightly decreases with further increasing temperature and crosses the gel- gel transition line at $54^{\circ} \mathrm{C}$, in good agreement with the rheological data (see Figure S1 in Supplementary information). The reduction in the effective volume fraction of micelles with increasing particle size at elevated temperatures is an indication of decreasing micelle number density. For the irradiated sample, the first transition occurs at $34{ }^{\circ} \mathrm{C}$ as shown in both scattering and rheological data, $6{ }^{\circ} \mathrm{C}$ higher than that of the unirradiated sample. This delayed gel formation is attributed to the higher LCST transition of the thermosensitive block after the removal of $o$-nitrobenzyl groups. The micelle 
volume fraction of the irradiated sample according to the model fitting decreases to the critical value at $43{ }^{\circ} \mathrm{C}, 11^{\circ} \mathrm{C}$ lower than in the unirradiated sample. However, the gel-sol transition for the irradiated sample observed in the rheological measurements take place at $52{ }^{\circ} \mathrm{C}, 9{ }^{\circ} \mathrm{C}$ higher than the expected temperature in terms of volume fraction. Various studies have shown that other factors such as interactions and penetration between the micellar corona, particle shape, arrangement and size distribution collectively determine the rheological properties besides packing volume fraction [36-38]. Starting at $43{ }^{\circ} \mathrm{C}$, we observe a dramatic increase in scattering intensity and change in slope of curves in the low q region for the irradiated sample as shown in the right graph of Figure 5, possibly signifying morphological transition that needs further investigation. Moreover, a significant broader size distribution in the irradiated sample than in the unirradiated one is also observed in terms of fitting (Tables S2 and S2 in SI). These two factors could cause the unexpectedly high gel-sol transition temperature in the irradiated sample.

Unimer insertion and micelle fusion are two pathways for micellar growth [39]. The scaling analysis suggests that the dominant structural evolution mechanism depends on how far the system is from equilibrium. Fusion is the prevalent growth mechanism when the system is far from equilibrium whereas the unimer insertion is preferred near equilibrium. ${ }^{40}$ In our study, we are able to calculate micelle number density through $n=\varnothing / v$, where $\phi$ and $v$ are micellar volume fraction and volume of a single particle respectively. The micelle number density reduces by a factor of 4 when the temperature was raised from 27 to $43{ }^{\circ} \mathrm{C}$ for both samples as shown in Figure 8, which indicates that the micellar growth follows fusion mechanism given that unimer insertion should not alter the number density. On the other hand, fusion alone should not result in dramatic change in micelle volume fraction. In the temperature range of 27 to $35^{\circ} \mathrm{C}$, the increase of volume fraction suggests that fusion is accompanied by unimer insertion in micelle growth. Above $35^{\circ} \mathrm{C}$, the particle volume fraction reduces (see Figure 7) and particle dimension grows slowly (Figure 6), both of which indicate the unimer insertion slows down. However, the micelle number density continues to drop between 35 and $43{ }^{\circ} \mathrm{C}$ (see Figure 8) especially for the irradiated sample, which implies that fusion mechanism is dominant at this stage. This agrees with the theoretical calculation: with micelles becoming denser, penetration of free unimers through the high polymer concentration region is energetically unfavorable [40]. The micellar growth obviously slows down beyond $43^{\circ} \mathrm{C}$ for both unirradiated and irradiated samples according to Figure 8. 


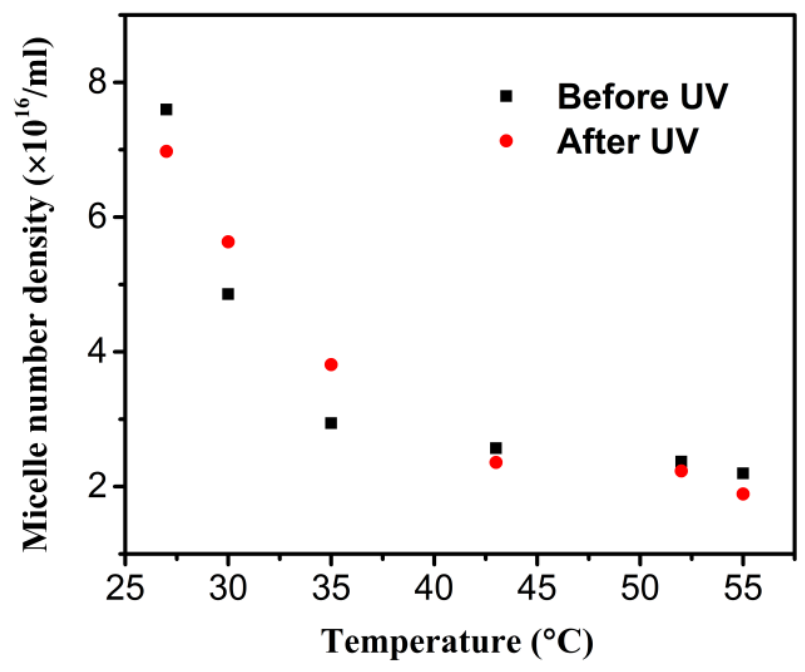

Figure 8. Number density of the micelles for aqueous solutions of PEO- $b$-P(TEGEA-co-NBA) (घ) and PEO- $b$-P(TEGEA-co-AA) formed from PEO-b-P(TEGEA-co-NBA) after UV irradiation $(\bullet)$ at $25 \mathrm{wt} \%$.

Further structural information of the micellar structure was obtained using material balance calculation, which was first used for analyzing scattering data of Pluronic P85-water system [18]. In the calculation, both EO monomer units and $\mathrm{D}_{2} \mathrm{O}$ are allowed to exist in the core and shell while TEGEA component only exists in the core region in order to solve four unknown parameters: aggregation number, fraction of $\mathrm{EO}$ monomer in the core, and numbers of $\mathrm{D}_{2} \mathrm{O}$ molecules in the core and shell via a group of four linear equations (3)-(6) described in the Methods Section.

Figure 9 displays the aggregation number of individual micelles and fraction of EO monomer units in the core as a function of temperature. We notice that the calculated results at $27^{\circ} \mathrm{C}$ and $30{ }^{\circ} \mathrm{C}$ for both unirradiated and irradiated samples are not reasonable albeit the scattering data at these temperatures can be fitted reasonably well using the spherical core-shell model. This can be ascribed to the deviation from our assumption that the TEGEA component only exists in the core. Both TEGEA and PEO monomers could be present in both core and corona regions during the early stages of micelle formation. Thus, the calculated results at $27^{\circ} \mathrm{C}$ and $30{ }^{\circ} \mathrm{C}$ are not included in the graphs. The unirradiated sample exhibits a monotonically increase in the 
aggregation number as increasing temperature. On the contrary, the slight decrease at $55{ }^{\circ} \mathrm{C}$ is observed in the irradiated sample. In addition, we notice that considerable amount of EO monomer units exist in the core region for both samples as displayed by the right graph of Figure 9. It is ascribed to progressively high chain density toward the center of micelles. Similar SLDs of EO concentration and TEGEA (Table 1) leads to part of EO segments in the corona is included in the core area in the model fitting. Interestingly the irradiated sample exhibits a smaller fraction of EO monomer units in the micelle core than in the unirradiated sample. This seemingly contradicts our previous claim of increasing hydrophilicity upon the removal of light sensitive group. Presumably RCOOH groups on the same or different chains tend to associate in aqueous solutions at elevated temperatures driven by hydrogen-bond formation and hydrophobic interactions, which has been reported by theoretical calculation and experiments [41-43]. The formation of associated $\mathrm{RCOOH}$ groups and hydrophobic interactions drive EO monomer and water molecules out of the core region. This is further evidenced by the water volume fraction in the micelle core as presented in Figure 10. The water volume fraction in the micelle core of the irradiated sample gradually declines to a negligible value with increasing temperature whereas it remains for the unirradiated sample in the measured temperature range. Given the growing number of the monomer units in the core, the solubility of the thermosensitive component in water becomes progressively worse with increasing temperature in both samples. The chain dehydration also takes place in the corona where PEO is the major component, reflecting reduced miscibility between PEO chains and water at elevated temperatures [44-47]. We believe that the loss of water in both core and corona of the micelles with increasing temperature leads to the second gel-mobile solution transition [4]. 

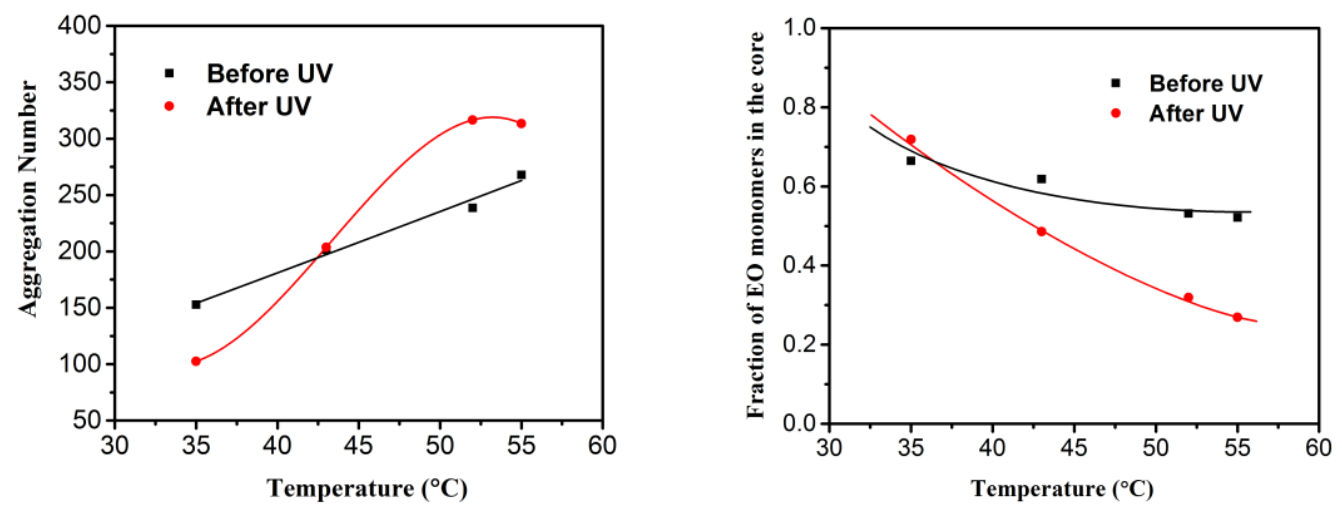

Figure 9. Variation of the aggregation number and fraction of EO monomer in the core region as a function temperature for the samples before and after UV exposure at $25 \mathrm{wt} \%$. The solid lines are included as a guide to the eye.
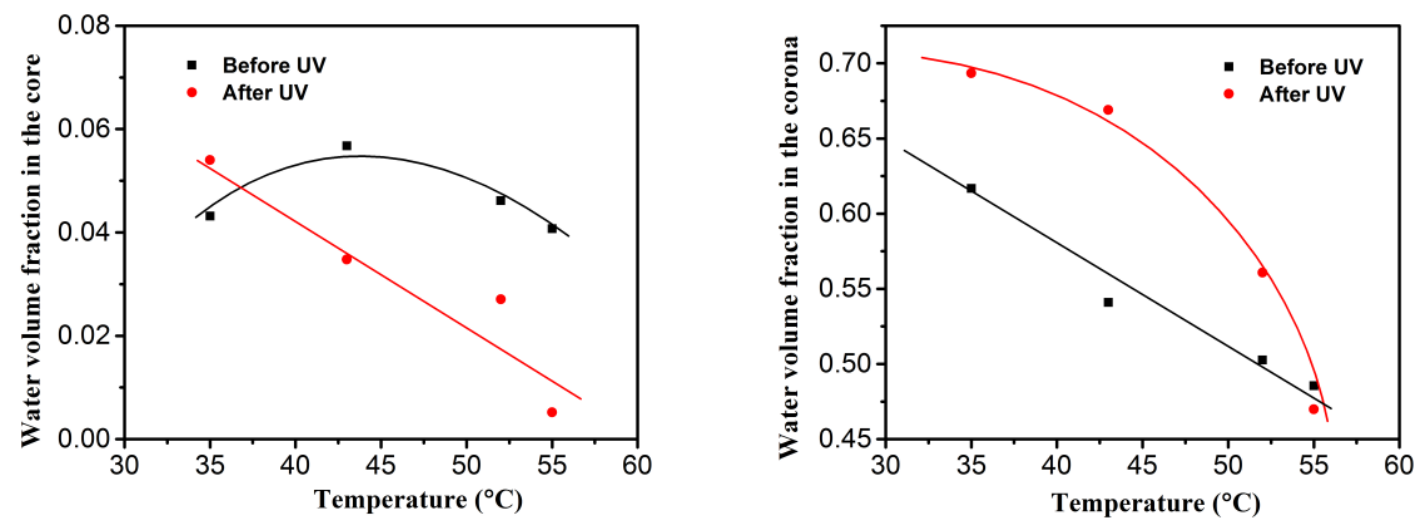

Figure 10. Variation of $\mathrm{D}_{2} \mathrm{O}$ volume fraction in the core (left) and corona (right) regions in both unirradiated and irradiated samples at $25 \mathrm{wt} \%$ as a function of increasing temperature. The solid lines are included as a guide to the eye.

\section{Summary}

In this work, we report a detailed SANS investigation on the micellization and gelation of PEO- $b$-P(TEGEA-co-NBA) as a function of temperature and concentration in aqueous solutions before and after the photocleavage of $o$-nitrobenzyl groups in the thermosensitive block. In dilute solutions, the unimers are observed when the temperature is below the LCST of the thermosensitive block. The chains collapse and form spherical micelles when the temperature reaches the LCST of the thermosensitive block. The removal of the $o$-nitrobenzyl group result in 
more expanded conformations and a higher micellization temperature due to the repulsive interactions between the ionized segments.

The variation of volume fraction of micelles in $25 \mathrm{wt} \%$ solution determined by the scattering data correlates well with the dynamic rheological data for the unirradiated sample. The discrepancy at the second transition temperature for the UV irradiated sample is likely ascribed to the broad micelle size distribution and morphological transition. Moreover, the variation of micelle number density as a function of increasing temperature combined with that of particle volume fraction suggests that micelle growth follows both unimer insertion and fusion mechanisms at the early stage of micelle formation. Water becomes a progressively worse solvent for both blocks with increasing temperature, as suggested by the distribution of water in the micelles. Dehydration with increasing temperature due to the breakup of hydrogen bonds between polymer chains and water result in the second gel-mobile solution transition.

\section{Acknowledgements}

This research used resources at the High Flux Isotope Reactor, a DOE Office of Science User Facility operated by the Oak Ridge National Laboratory. B.Z. thanks NSF for the support (DMR1206385 and -1607076).

\section{Appendix A. Supplementary Data.}

Supplementary data related to this article can be found at http://dx.doi.org/10.1016/

\section{References}

[1] E. S. Gil; S. M. Hudson, , Prog Polym Sci, 2004, 29, 1173-1222.

[2] T. G. O'Lenick, X. G. Jiang, B. Zhao, Langmuir, 2010, 26, 8787-8796.

[3] X. Jiang, C. A. Lavender, J. W. Woodcock, B. Zhao, Macromolecules, 2008, 41, 2632-2643.

[4] X. G. Jiang, S. Jin,Q. X. Zhong,M. D.Dadmun,B. Zhao, Macromolecules 2009, 42, 8468-8476.

[5] B. Jeong, S. W. Kim, Y. H. Bae, Adv. Drug Deliver. Rev., 2002, 54, 37-51.

[6] C. L. He, S. W. Kim, D. S. Lee, J. Control Release 2008, 127, 189-207.

[7] T. G. O'Lenick, N. X. Jin, J. W. Woodcock, B. Zhao, J. Phys. Chem. B 2011, 115, 2870-2881.

[8] I. W. Hamley, V. Castelletto, Prog. Polym. Sci., 2004, 29, 909-948.

[9] J. S. Pedersen, Adv. Colloid Interfac., 1997, 70, 171-210.

[10] R. P. Redouane Borsali, Soft Matter Characterization. Springer Netherlands: 2008, Vol. 1.

[11] J. S. Pedersen, M. C. Gerstenberg, Macromolecules, 1996, 29, 1363-1365.

[12] J. S. Pedersen, J. Appl. Crystallogr., 2000, 33, 637-640.

[13] J. S. Pedersen, J. Chem. Phys., 2001, 114, 2839-2846. 
[14] M. C. Gerstenberg, J. S. Pedersen, Langmuir, 2001, 17, 7040-7046.

[15] C. Svaneborg, J. S. Pedersen, Macromolecules, 2002, 35, 1028-1037.

[16] J. S. Pedersen, M. C. Gerstenberg, Colloid Surface A, 2003, 213, 175-187.

[17] T. P. Lodge, X. Xu, C. Y. Ryu, I. W. Hamley, J. P. A. Fairclough, A. J. Ryan, J. S. Pedersen, Macromolecules, 1996, 29, 5955-5964.

[18] B. Hammouda, Eur. Polym. J., 2010, 46, 2275-2281.

[19] S. L. Martin, L. L. He, F. Meilleur, R. H. Guenther, T. L.Sit, S. A. Lommel, W. T. Heller, Arch. Virol., 2013, 158, 1661-1669.

[20] G. D. Wignall, K. C. Littrell, W. T. Heller, Y. B. Melnichenko, K. M. Bailey, G. W. Lynn, D. A. Myles, V. S. Urban, M. V. Buchanan, D. L. Selby, P. D. Butler, J. Appl. Crystallogr., 2012, 45, 990-998.

[21] G. D. Wignall, F. S. Bates, J. Appl. Crystallogr. 1987, 20, 28-40.

[22] S. R. Kline, J. Appl. Crystallogr., 2006, 39, 895-900.

[23] B., Hammouda, Probing nanoscale structures-the SANS toolbox. 2009.

[24] S. H. Chen, T. L. Lin, Colloidal Solutions, 1987; Vol. 23.

[25] J. K. Percus, G. J. Yevick, Phys. Rev., 1958, 110, 1-13.

[26] D. J. Kinning, E. L. Thomas, Macromolecules, 1984, 17, 1712-1718.

[27] B. Hammouda, D. L. Ho, S. Kline, Macromolecules, 2004, 37, 6932-6937.

[28] B. Hammouda, Adv. Polym. Sci., 1993, 106, 87-133.

[29] K. Mortensen, W. Brown, B. Norden, Phys. Rev. Lett., 1992, 68, 2340-2343.

[30] K. E. B. Doncom, A. Pitto-Barry, H. Willcock, A. Lu, B. E. McKenzie, N. Kirby, R. K. O'Reilly, Soft Matter, 2015, 11, 3666-3676.

[31] J. S. Higgins, H. C. Benoit, Polymers and Neutron Scattering. Oxford Science Publications: New York, 1994; p 436.

[32] J. Lu, F. S. Bates, T. P. Lodge, Macromolecules, 2015, 48, 2667-2676.

[33] S. Y. Choi, F. S. Bates, T. P. Lodge, J. Phys. Chem. B, 2009, 113, 13840-13848.

[34] G. M. Mao, S. Sukumaran, G. Beaucage, M. L. Saboungi, P. Thiyagarajan, Macromolecules, 2001, 34, 4666-4666.

[35] I. W. Hamley, S. M. Mai, A. J. Ryan, J. P. A. Fairclough, C. Booth, Chem. Chem. Phys., 2001, 3, 2972-2980.

[36] S. M. Olhero, J. M. F. Ferreira, Powder Technol., 2004, 139, 69-75.

[37] P. F. Luckham, M. A. Ukeje, J. Colloid. Interf. Sci. 1999, 220, 347-356.

[38] S. Mueller, E. W. Llewellin, H. M. Mader, P. Roy. Soc. a-Math Phy. 2010, 466, 1201-1228.

[39] N. Hadjichristidis, S. Pispas, G. Floudas, Block Copolymers: Synthetic Strategies, Physical Properties, and Applications. John Wiley \& Sons: 2002.

[40] E. E. Dormidontova, Macromolecules, 1999, 32 (22), 7630-7644.

[41] K. Yamamoto, N. Nishi, J. Am. Chem. Soc., 1990, 112, 549-558.

[42] G. Nemethy, I. Z. Steinberg, I. Z.; H. A. Scheraga, Biopolymers, 1963, 1, 43-69.

[43] V. Doan, R. Koppe, P. H. Kasai, J. Am. Chem. Soc., 1997, 119, 9810-9815.

[44] L. Yang, P. Alexandridis, D. C. Steytler, M. J. Kositza, J. F. Holzwarth, Langmuir, 2000, 16, 8555-8561.

[45] P. Alexandridis, J. F. Holzwarth, T. A.Hatton, Macromolecules, 1994, 27, 2414-2425.

[46] P. Linse, Macromolecules, 1994, 27, 6404-6417.

[47] P. Linse, Macromolecules, 1993, 26, 4437-4449. 